\title{
Análise de requisitos de identificação e autorização para dispositivos e gateways de borda em IIoT
}

\author{
Sergio Henrique Silva ${ }^{1}$, Charles C. Miers ${ }^{1}$ \\ ${ }^{1}$ Programa de Pós-Graduação em Computação Aplicada (PPGCA) \\ Universidade do Estado de Santa Catarina (UDESC) \\ sergio.hs@edu.udesc.br, charles.miers@udesc.br
}

\begin{abstract}
Resumo. O uso de dispositivos inteligentes para as mais diversas atividades humanas fez emergir o conceito como IoT e também de IIoT quando estes dispositivos são aplicados a indústria. A gestão de identidades e segurança em ambientes IIoT tem limitações e especificidades que resultam em complexidade. O trabalho tem como objetivo identificar e estabelecer critérios que podem mensurar a aplicabilidade de uma abordagem de gestão de identidades em dispositivos aos cenários IIoT. Propõe-se uma análise de requisitos e da arquitetura em IIoT para a identificação de critérios, que posteriormente serão avaliados por experimentos para serem então estabelecidos como válidos.
\end{abstract}

\section{Introdução e motivação}

A Industrial Internet of Things (IIoT) pode ser definida como um sistema que conecta e integra sistemas de controle industrial com sistemas empresariais, e também como um conjunto de processos de negócios e análises, usando sistemas de controle industrial que contêm sensores e atuadores [IIC, 2019b]. Estes sistemas também são normalmente de considerável complexidade.

O Industrial Internet Consortium (IIC) defende como uma das arquiteturas referenciais em IIoT, a arquitetura de três camadas [IIC, 2019a]: (i) Borda: formada pelos dispositivos físicos, sensores, atuadores e controladores que coletam dados ou executam funções específicas, dispositivos marcados pela pouca disponibilidade de recursos; (ii) Plataforma de Serviço: camada formada pela infraestrutura de comunicação e controle dos dispositivos na rede. A mesma recebe, processa e encaminha comandos de controle da camada corporativa para a camada de borda; e (iii) Corporativa: recebe dados advindos da borda e da camada de plataforma, também emitindo comandos de controle para a camada da plataforma e camada de borda.

Em um cenário de IIoT, no qual existem dispositivos de borda em larga escala com restrições de recursos como processamento e memória os procedimentos de segurança, como a infraestrutura de autenticação devem ser planejados levando em conta as peculiaridades deste cenário [Al-Sharekh and Al-Shqeerat, 2019]. Contudo, a IIoT também necessita de segurança visto que uma falha pode comprometer desde falhas pequenas na operação até riscos a integridade física de seres humanos. Dispositivos não autorizados podem comprometer o controle e a continuidade das operações de uma organização. Diante deste quadro, este trabalho apresenta uma proposta de critérios para análise de abordagens em infraestruturas de autenticação e sua aplicabilidade aos contextos de IIoT. 
Na Seção 2 enuncia-se uma introdução a conceitos básicos em identificação e autorização, também sua relação com os cenários de IIoT bem como suas classificações. A Seção 3 introduz os trabalhos relacionados a segurança da informação, com ênfase para autenticação e autorização em dispositivos IIoT. Na Seção 4 propõe-se critérios para análise da aplicabilidade das diversas abordagens de autenticação e autorização.

\section{Identificação e autorização em dispositivos}

A autorização e autenticação são operações que fornecem o controle de acesso em sistemas distribuídos e usualmente fazem parte de uma infraestrutura de autorização e autenticação (IAA) que faz a gestão de identidades, i.e., Identity Manager (IdM). ITU, 2009 afirma que um IdM pode ser definido como um conjunto de tecnologias e processos usados para validar a identidade de uma entidade (um usuário ou um dispositivo) a fim de utilizar um recurso computacional.

Um sistema de IdM é composto pelas entidades: (i) usuário ou dispositivo que utiliza um serviço fornecido por um provedor de serviços; (ii) provedor de identidades Identity Provider (IdP), responsável por manter a base de dados dos usuários do domínio e verificar suas credenciais (autenticar usuário ou dispositivo); e (iii) provedor de serviços Service Provider (SP), que oferece recursos ou serviços aos usuários [Wangham et al., 2010]. A autenticação é tratada de forma diferente para usuários e para dispositivos em suas operações. Assim, é necessária uma abordagem específica para as abordagens de autenticação em dispositivos. Portanto, neste trabalho, trata-se a autenticação e autorização de dispositivos de maneira transversal em relação a IoT e IIoT.

\subsection{Padrões em identificação e autorização em dispositivos}

A padronização é um enfoque importante para concretização de ambientes heterodoxos como IIoT. Observa-se um esforço das entidades de padronização de países pelo mundo e de entidades internacionais no intuito de estabelecer padrões. Em relação às abordagens, métodos e boas práticas em segurança, autenticação e autorização relaciona-se padrões advindos das principais instituições de regulação como International Organization for Standardization (ISO), International Telecommunication Union (ITU) e National Institute of Standards and Technology (NIST). A Tabela 1 lista os principais padrões relacionados a segurança de IIoT.

Tabela 1. Padrões relacionados a lloT: autenticação/autorização de dispositivos.

\begin{tabular}{l|l|l}
\hline Orgão & Identificação & Título \\
\hline IEEE & IEEE P1451-99 & $\begin{array}{l}\text { Standard for Harmonisation of Internet of Things (IoT) Devi- } \\
\text { ces and Systems }\end{array}$ \\
\hline IEEE & IEEE 802.15.4 & IEEE Standard for Low-Rate Wireless Networks \\
\hline ISO/IEC & $\begin{array}{l}\text { ISO/IEC } \\
\text { JTC }\end{array}$ & $\begin{array}{l}\text { Security techniques - Extension to 27001/27002 for Guideli- } \\
\text { nes for privacy information management }\end{array}$ \\
\hline ISO/IEC & $\begin{array}{l}\text { ISO/IEC } \\
\text { JTC }\end{array}$ & $\begin{array}{l}\text { Information technology - Security techniques - Information } \\
\text { security risk management }\end{array}$ \\
\hline ITU-T & $\begin{array}{l}\text { ITU-T X.1361 } \\
(09 / 2018)\end{array}$ & $\begin{array}{l}\text { Security framework for the Internet of things based on the ga- } \\
\text { teway model environments }\end{array}$ \\
\hline NIST & NIST 8259A & IoT Device Cybersecurity Capability Core Baseline \\
\hline
\end{tabular}


Na Tabela 1 os itens são enquadrados conforme a relação de [Labib et al., 2019] e de acordo com sua aplicabilidade em IIoT, fazendo correlações com a infraestrutura, segurança, autenticação e autorização de dispositivos.

\subsection{Taxonomias em identificação e autorização em dispositivos IIoT}

$\mathrm{Na}$ literatura de taxonomias em autenticação e autorização de dispositivos, observa-se iniciativas em esquematizar as operações e abordagens neste processo. As principais abordagens identificadas que possuem aproximação com o enfoque deste trabalho:

- [El-hajj et al., 2019] : Os autores fornecem uma visão abrangente e atualizada do campo de autenticação em Internet of Things (IoT). Disponibilizam um resumo de uma variedade de protocolos de autenticação propostos na literatura revisada no trabalho. Utilizam uma classificação multicritério comparando e avaliando os protocolos de autenticação propostos e mostrando seus pontos fortes e fracos;

- [Lopez et al., 2019]: Elabora-se uma taxonomia de sistemas que fornecem soluções conjuntas para problemas de autenticação e autorização ou soluções para apenas um dos problemas. O trabalho é focado em sistemas já desenvolvidos, em desenvolvimento ou em sua fase experimental, excluindo propostas teóricas.

- [Pulkkis et al., 2006]: Discute e classifica a autenticação de usuários com base em diferentes taxonomias, classificadas em relação a: identificação de usuário, método de autenticação, qualidade da autenticação, complexidade da autenticação e escopo da autenticação.

\subsection{Trabalhos relacionados}

A segurança da informação é vital para qualquer contexto computacional, mas as especificidades dos contextos de IoT como a escassez de recursos computacionais nos dispositivos faz os desafios na disponibilidade de serviços computacionais tomarem contornos específicos. Em surveys relacionando segurança da informação, IoT e IIoT como [Jurcut et al., 2020] e [Sengupta, 2019] faz-se uma relação de alguns trabalhos relevantes em segurança da informação, autenticação e autorização em IoT.

Ammar et al., 2018 pesquisou segurança e privacidade em frameworks em IoT, fazendo comparações nos quesitos de autenticação, controle de acesso, comunicação, criptografia de segurança. A pesquisa contribui com critérios de análise de segurança em frameworks IoT.

Yang et al., 2017 realizou um estudo sobre soluções de segurança levando em conta as limitações dos dispositivos IoT, fazendo uma classificação de ataques em IoT, autenticação e controle de acesso, mecanismos e análise de segurança em diferentes camadas do modelo OSI.

Lin et al., 2017 apresentou uma visão geral da arquitetura dos sistemas de proteção em IoT, tecnologias habilitadoras, segurança e questões de privacidade na integração da IoT com a Edge ou Fog computing. Neste trabalho, IoT é apresentada como uma infraestrutura de rede que compreende dispositivos e sistemas para compartilhamento, análise e gerenciamento de recursos. A confidencialidade, integridade, disponibilidade, identificação, autenticação, privacidade e confiabilidade são discutidas como recursos de segurança da IoT. 
Nos trabalhos relatados, pode-se observar diversos tipos de experimentos tratando de autenticação e autorização em IoT ou IIoT na literatura. Em conseguinte este trabalho dedica-se a traçar critérios objetivos para análise de arquiteturas ou implementações como as que foram relacionadas de forma que se possa aferir sua aplicabilidade aos cenários IoT ou IIoT.

\section{Segurança, autenticação e autorização em dispositivos}

Dentre os trabalhos analisados, El-hajj et al., 2019 apresentam uma taxonomia de esquemas de autenticação IoT (aplicável a IIoT) usando diversos critérios selecionados com base nas semelhanças e nas principais características dos esquemas relacionados pelos autores em seu trabalho. Esses critérios são relacionados na Figura 1.

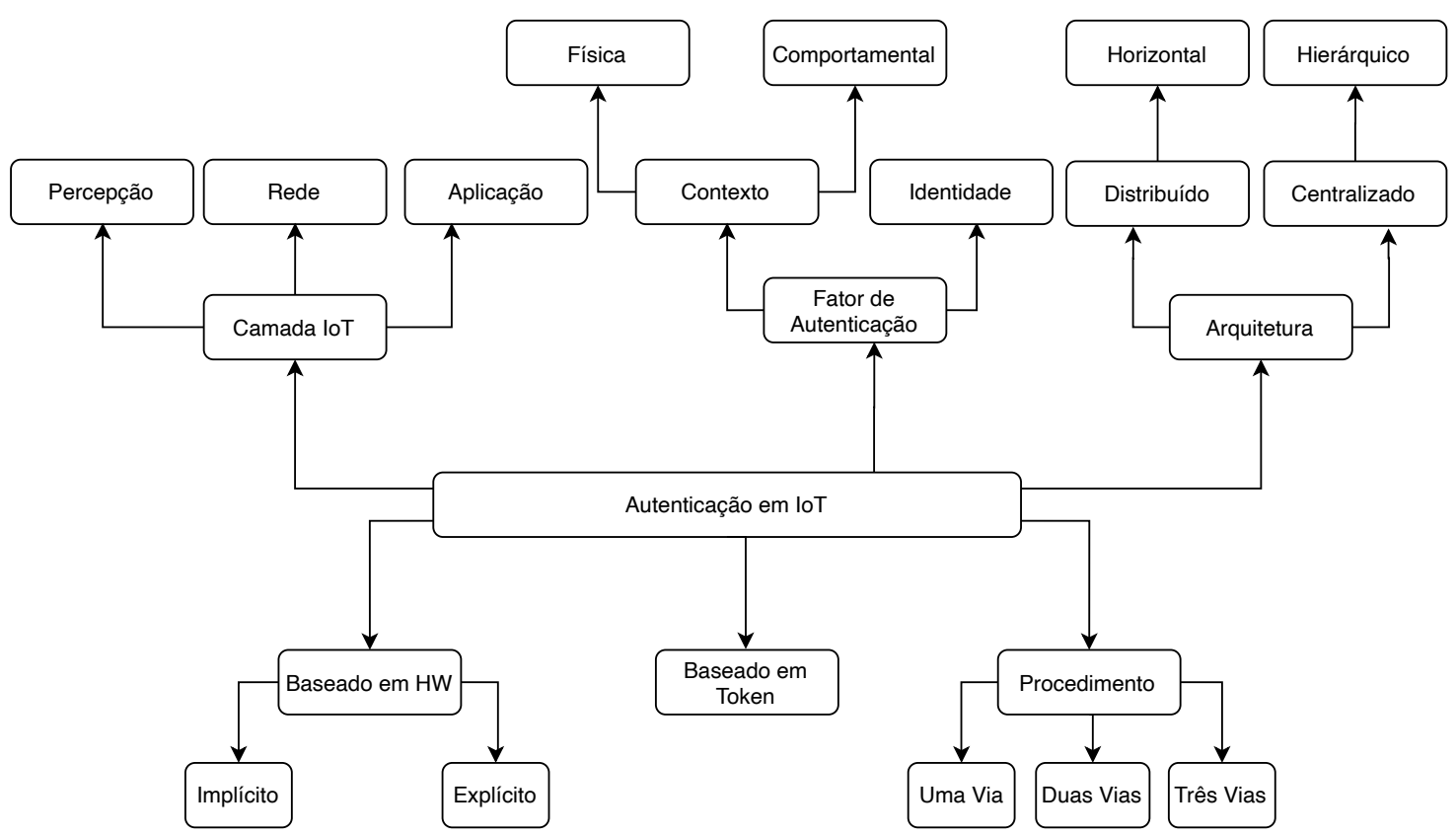

Figura 1. Taxonomia de autenticação loT. Adaptado de: [EI-hajj et al., 2019]

Na classificação (Figura 1) pode-se observar diferentes dimensões relacionadas a autenticação de dispositivos. Este arranjo permite analisar cada implementação ou proposta por múltiplos fatores, relacionando estas implementações aos requisitos de IIoT. Apesar da intrínseca ligação entre a IoT e IIoT, os requisitos de ambas tem alguns pontos de divergência, que tornam abordagens de autenticação aplicáveis ou não aplicáveis aos contextos de IIoT. Partindo-se uma classificação estruturada e dos requisitos dos ambientes IIoT, pode-se traçar os critérios da análise.

\section{Critérios para análise da autenticação e autorização em IoT e IIoT}

Este trabalho tem como objetivo a análise das abordagens de autenticação de dispositivos inteligentes, especialmente em ambientes IIoT. Neste sentido, analisando os trabalhos listados nas Seções 2.1 e 3, foram definidos quatro critérios iniciais para conduzir esta análise:

1. Adequação aos padrões estabelecidos: As abordagens de gestão de identidades analisadas devem seguir os padrões estabelecidos pelas entidades padronizadoras nacionais e 
internacionais. Estas abordagens devem seguir os protocolos derivados destes padrões como o caso do Wi-Fi Protected Access (WPA) em referência ao padrão IEEE 802.11. Este critério ainda analisa se a abordagem de utiliza sistemas de autenticação como openID, se tem suporte para soluções de autenticação federada com protocolos ad hoc como SAML no provimento de identidades.

2. Escalabilidade: Verificar a adequação dos processos de autenticação e autorização à escala industrial, como elaborado em [Consel and Kabac, 2017] que relaciona diversos casos do uso de dispositivos de IoT em larga escala como o gerenciamento de vagas de estacionamento em cidades, monitoramento de sistemas de transporte nas cidades e monitoramento da cadeia de produção de diversos setores industriais. Neste critério serão avaliadas a gestão das identidades pela sua garantia de escalabilidade e disponibilidade para contextos de utilização de dispositivos em grande escala.

3. Uso de técnicas de computação de borda (Edge Computing): A computação de borda, também denominada de Edge Computing ou Fog Computing [Lin et al., 2017]. Neste critério as abordagens de gestão de identidade serão julgadas de acordo com a possibilidade de que o processamento de operações de autenticação e autorização possam ser delegadas a outros dispositivos como um serviço.

4. Comunicação Machine to Machine (M2M): Esfahani et al., 2019 relacionam a comunicação M2M como uma das principais características inerentes a IIoT e a Indústria 4.0. Este tipo de comunicação pressupõe que exista a comunicação direta entre os dispositivos e, a partir disso, se torna viável o disparo de ações automáticas entre os equipamentos da camada de borda, e.g., sensores e atuadores. Este critério nivelará as abordagens de autenticação levando em consideração a sua aplicabilidade em cenários de comunicação direta entre dispositivos como M2M.

\section{Considerações e trabalhos futuros}

Apesar de IoT e IIoT terem como principal característica a disponibilidade de dispositivos inteligentes conectados, a IoT é direcionada ao consumidor final, tem sensores de baixa ou média capacidade e tem baixo risco na utilização. Já a IIoT é empregada em cadeias de suprimentos completas, em larga escala, usando sensores mais precisos em ambientes com riscos potenciais. Neste cenário, a proposta visa atender aos padrões de segurança, além dos requisitos específicos de IIoT

As próximas atividades a serem desenvolvidas nesta pesquisa são: (i) Identificar abordagens estabelecidas ou experimentais em gestão de identidade para os experimentos; (ii) Revisitar e, se necessário, aprimorar a lista de critérios de avaliação; e (iii) Analisar os dados coletados, relaciona-los com os padrões e critérios definidos e, em caso de hipotético sucesso da avaliação, aferir a adequação das abordagens de autenticação e autorização a IIoT.

Agradecimentos: Os autores agradecem o apoio do LabP2D/UDESC e da FAPESC.

\section{Referências}

Al-Sharekh, S. I. and Al-Shqeerat, K. H. A. (2019). Security challenges and limitations in iot environments. IJCSNS International Journal of Computer Science and Network Security. 
Ammar, M., Russello, G., and Crispo, B. (2018). Internet of things: A survey on the security of iot frameworks. Journal of Information Security and Applications, 38:8 27.

Consel, C. and Kabac, M. (2017). Internet of things: From small- to large-scale orchestration. 2017 IEEE 37th International Conference on Distributed Computing Systems (ICDCS), pages 1748-1755.

El-hajj, M., Fadlallah, A., Maroun, C., and Serhrouchni, A. (2019). A survey of internet of things (iot) authentication schemes. Sensors, 19.

Esfahani, A., Mantas, G., Matischek, R., Saghezchi, F. B., Rodriguez, J., Bicaku, A., Maksuti, S., Tauber, M. G., Schmittner, C., and Bastos, J. (2019). A lightweight authentication mechanism for $\mathrm{m} 2 \mathrm{~m}$ communications in industrial iot environment. IEEE Internet of Things Journal, 6(1):288-296.

IIC, I. I. C. (2019a). The industrial internet of things: Reference architecture.

IIC, I. I. C. (2019b). The industrial internet of things vocabulary technical report.

ITU, I. T. U. (2009). Ngn identity management framework. Recommendation ITU-T Y.2720, 01 .

Jurcut, A., Niculcea, T., Ranaweera, P., and Le-Khac, N. (2020). Security considerations for internet of things: A survey. Journal of Network and Computer Applications, page 19.

Labib, N. S., Brust, M. R., Danoy, G., and Bouvry, P. (2019). Trustworthiness in iot - a standards gap analysis on security, data protection and privacy. IEEE Conference on Standards for Communications and Networking (CSCN).

Lin, J., Yu, W., Zhang, N., Yang, X., Zhang, H., and Zhao, W. (2017). A survey on internet of things: Architecture, enabling technologies, security and privacy, and applications. IEEE Internet of Things Journal, 4:1125-1142.

Lopez, J., Montenegro, J. A., Oppliger, R., and Pernul, G. (2019). On a taxonomy of systems for authentication and/or authorization services. TERENA Networking Conference.

Pulkkis, G., Grahn, K., and Karlsson, J. (2006). Taxonomies of user-authentication methods in computer networks.

Sengupta, J. (2019). A comprehensive survey on attacks, security issues and blockchain solutions for iot and iiot. Journal of Network and Computer Applications.

Wangham, M. S., de Mello, E. R., da Silva Boger, D., Guerios, M., and da Silva Fraga, J. (2010). Gerenciamento de identidades federadas. GT-STCFed-RNP, 2010.

Yang, Y., Wu, L., Yin, G., Li, L., and Zhao, H. (2017). A survey on security and privacy issues in internet-of-things. IEEE Internet of Things Journal, 4(5):1250-1258. 